\title{
Extraction of phenolic compounds from agro-industrial by-products by fungal fermentation with potential use as additives for meat and meat products. A review
}

\author{
Extracción de compuestos fenólicos de subproductos agroindustriales por fermentación fúngica con uso \\ potencial como aditivos para carne y productos cárnicos. Revisión
}

\begin{abstract}
Rey David Vargas-Sánchez ${ }^{1, * *}$, Brisa del Mar Torres-Martínez ${ }^{1}$, Gastón Ramón Torrescano-Urrutia ${ }^{1}$, Armida SánchezEscalante ${ }^{1}$, Martín Esqueda ${ }^{2}$

Coordinación de Tecnología de Alimentos de Origen Animal, Centro de Investigación en Alimentación y Desarrollo, A.C. Carretera Gustavo Enrique Astiazarán Rosas, No. 46, Col. La Victoria, CP 83304, Hermosillo, Sonora, México.

2 Coordinación de Tecnología de Alimentos de Origen Vegetal, Centro de Investigación en Alimentación y Desarrollo, A.C. Carretera Gustavo Enrique Astiazarán Rosas, No. 46, Col. La Victoria, CP 83304, Hermosillo, Sonora, México.

\# Cátedras CONACYT-CIAD, A.C.
\end{abstract}

\section{ABSTRACT}

The present manuscript reviews the findings of different research studies that evaluate the use of fungal fermentation-assisted extraction, in solid-state fermentation (SSF) and submerged culture fermentation (SCF) with agroindustrial residues as substrates, to obtain phenolic compounds with possible applications as food additives. Some agro-industrial by-products (peels, pulps and seeds) are an important source of phenolic acids such as $p$-coumaric, p-hydroxybenzoic, chlorogenic, cinnamic, ferulic, gallic, protocatechuic, rosmarinic, syringic, and vanillic acids and flavonoids (apigenin, chrysin, (+)-catechin, kaempferol, myricetin, quercetin, rutin, hesperetin, and naringin). In addition, the utilization of these by-products as substrates in SSF and SCF allowed obtaining phenolic compounds with antioxidant and antimicrobial activities. Thus, fungal fermentationassisted extraction provides a potential alternative to obtain natural additives for meat and meat products industry.

Keywords: Mushroom, Fermentation, Compound extraction, Food additives

\section{RESUMEN}

El presente manuscrito revisa los hallazgos de diferentes estudios de investigación que evalúan el uso de la extracción-asistida por fermentación fúngica, en medio sólido (SSF) y cultivo sumergido (SCF) con subproductos agroindustriales como sustratos, para obtener compuestos fenólicos con posible uso como aditivos alimentarios. Algunos subproductos agroindustriales (pulpas, cáscaras y semillas) son una fuente importante de ácidos fenólicos como $p$-cumárico, $p$-hidroxibenzoico, clorogénico, cinámico, ferúlico, gálico, protocatecuico, rosmarínico, siríngico, y vanílico, y de flavonoides (apigenina, crisina, (+)-catequina, kaempferol, miricetina, quercetina, rutina, hesperetina y naringina). Además, la utilización de estos subproductos como sustratos en SSF y SCF permitió obtener compuestos fenólicos con actividad antioxidante y antimicrobiana. Por lo que, la extracción-asistida por fermentación fúngica proporciona una alternativa potencial para obtener aditivos naturales para la industria de la carne y productos cárnicos.

Palabras clave: Hongos, Fermentación, Extracción de compuestos, Aditivos alimentarios.

\section{INTRODUCTION}

Meat and meat products are considered an important source of many essential nutrients in the human diet, including lipids such as fatty acids (mono- or polyunsaturated) and proteins rich in essential amino acids such as isoleucine, leucine, lysine, methionine, phenylalanine, threonine, and valine, which are highly susceptible to oxidation processes (Hammad et al., 2020). Oxidative deterioration of lipids and proteins, and microbial growth are considered the main causes of quality loss in any type of meat or meat products leading to organoleptic and technological changes such as color, odor, flavor, appearance, and texture, as well as water holding capacity and water loss by cooking. In addition, both factors promote nutrient losses and toxic compound formation (Jiang and Xiong, 2016; Aziz and Karboune, 2018). However, the uncontrolled uses of antioxidant and antimicrobial additives to preserve meat and meat products is a practice that generate negative effects on consumer health; thus, strict regulations for their controlled used in foods has been promoted (Poljsak et al., 2013; Aziz and Karboune, 2018).

In previous investigations to reduce lipid and protein oxidation and microbial growth in meat and meat products, extracts rich in phytochemicals have been obtained from plants, herbs, and species, and used instead of synthetic preservatives (Jiang and Xiong, 2016). In addition, the reuse of agro-industrial by-products such as peel pomace and seeds offers an alternative source of additives with antioxidant and antimicrobial properties (Hernández-Carlos et al., 2019).

Moreover, several extraction methods have been developed to obtain bioactive compounds from agro-industrial by-products, such as conventional (maceration and 
hydrodistillation extraction) and unconventional (ultrasonic, microwave, supercritical fluid, and enzyme extraction) methods. These extraction methods in combination with a solvent system of different polarities, including water, acetone, ethanol, methanol, hexane, and petroleum ether, improve the types of compounds that can be extracted (Azmir et al., 2013; Hernández-Carlos et al., 2019). However, biotechnological methods such as fungal fermentation-assisted extraction (SSF and SCF) has been suggested as an additional alternative to obtain bioactive compounds from agro-industrial by-products (Papaspyridi et al., 2012; Santana-Méridas et al., 2012). In this review, a general description of the uses of fungal fermentation-assisted extraction (SSF and SCF) to obtain phenolic compounds from agro-industrial by-products, and their possible applications as food additives are discussed.

\section{Phenolic Compounds from Agro-Industrial By-Products}

The definition of food losses and waste could be associated with a reduction in the availability of food, a decrease in nutritional value and a deterioration in food safety, involving many players in food supply chains, such as farmers and processors. Furthermore, food losses could occur by accidental causes (intrinsic or extrinsic factors), and food waste occurs for reasons of negligence (FAO, 2017). The Mexican normative (NOM-251-SSA1-2009) defines food residue/byproduct as'waste from processed raw material'. In this regard, the food processing industry generates large amounts of byproducts, including pomace, husks, seeds, leaves, stems, and wood (Peanparkdee and Iwamoto, 2019; Rico et al., 2020). In some cases, these by-products are treated to decrease negative environmental impact, making them a useful product with the added benefits of solving a problem and generating additional income (Rico et al., 2020).

Agro-industrial by-products are commonly disposed of, used on-site or used off-site or after pre-treatment. These can be pre-treated by physicochemical (combustion, pyrolysis, and gasification) or biochemical (anaerobic digestion and fermentation) processes, to generate biodiesel and electricity or bio-alcohol and biogas, respectively. In addition, agroindustrial by-products can be pre-treated by bio-reduction to produce animal feed, and by chemical modifications, and by SSF and SCF to obtain bioactive compounds (SantanaMéridas et al., 2012). Thus, agro-industrial by-products are considered a rich source of bioactive compounds, including alkaloids, terpenoids, saponins, essential amino acids and fatty acids, minerals, carotenoids, vitamins, polysaccharides, and phenolic compounds like phenolic acids, and flavonoids (Wijngaard et al., 2012; Azmir et al., 2013; Peanparkdee and Iwamoto, 2019; Rico et al., 2020).

The major by-products of fruit processing are peel and seed, and in a minor proportion, pulp (Santana-Méridas et al., 2012). However, the extraction, identification and uses of phenolic compounds are widely investigated in commercial sectors such as the pharmaceutical, chemical, and food industries (Azmir et al., 2013; Santana-Méridas et al., 2012). In this context, table 1 compiled literature reports of these residues as an important source of phenolic acids, including peel (apple, potato, and tomato), pulp (avocado) and seeds (avocado, citrus, and tomato). In addition, table 2 demonstrate that by-products also are a significant source of flavones, flavonols, and flavanones compounds. It has been reported that phenolic compounds are present ubiquitously in all parts of plants such as wood, leaves, roots, and fruits (Vermerris and Nicholson, 2008; Rico et al., 2020). In this regard, these compounds are commonly trapped or bound to the dietary fiber of plant material, through hydrogen bonds between the phenol hydroxyl group (HO) of the phenolic component, hydrophobic interactions, and covalent bonds like ester bond between phenolic acids and polysaccharides (Quirós-Sauceda et al., 2011).

Chemical structure plays a key role in the bioactivity of phenolic compounds, which have been associated with several key factors such as $\mathrm{OH}$-group location in the benzene ring, the substitution patterns by the $\mathrm{OH}$-group (ortho-, meta-, para-, meta-tri-, vic-tri-), the presence of glycosylation, and double bounds in the benzene structure (Vermerris and Nicholson, 2008; Rico et al., 2020). However, the types of phenolic compounds obtained, and their bioactivity are closely associated with the extraction method employed (Azmir et al., 2013).

\section{Extraction Methods}

Phenolic compounds are widely found as a mixture of different components in a solid, and for extraction that are dispersed in a liquid phase, which allows their separation from the solid phase. This process is known as liquid-solid extraction, and to increase the diffusion rate of the solvent in the solute and yields, it is necessary to dry and reduce the particle size of the solid or plant material (fruits, leaves, stem, roots, wood, flowers or seeds) (Pinelo et al., 2007; Pronyk and Mazza, 2009; Orphanides et al., 2013).

Furthermore, several methods are frequently employed to obtained phenolic compounds, including rustic methods (extraction by cooking, percolation, and infusion), conventional methods (extraction by maceration, Soxhlet, and hydrodistillation) and unconventional methods, including enzymes-assisted extraction, microwave-assisted extraction, pressurized liquid-assisted extraction, supercritical fluids-assisted extraction, and ultrasound-assisted extraction (Wijngaard et al., 2012; Azmir et al., 2013). However, the solvent type, solvent-solid ratio, number of extractions, $\mathrm{pH}$, temperature, time, vacuum and fermentation process, among other conditions used, influences phenolic yields (Spigno et al., 2007; Ramírez-Rojo et al., 2018).

\section{Fungal Culture Fermentation}

SSF involves the fermentation of solids or semi-solids in the absence of water, where the substrate used to be the source of moisture to support microbial growth (Pandey, 2003; Castañeda-Casasola et al., 2018), while in SCF, microorganisms grow submerged with an excess of water and limited oxygen (Castañeda-Casasola et al., 2018). In this context, 
Table 1. Basic structure of phenolic acids identified in some agro-industrial by-products.

Tabla 1. Estructura básica de ácidos fenólicos identificados en algunos subproductos agroindustriales.

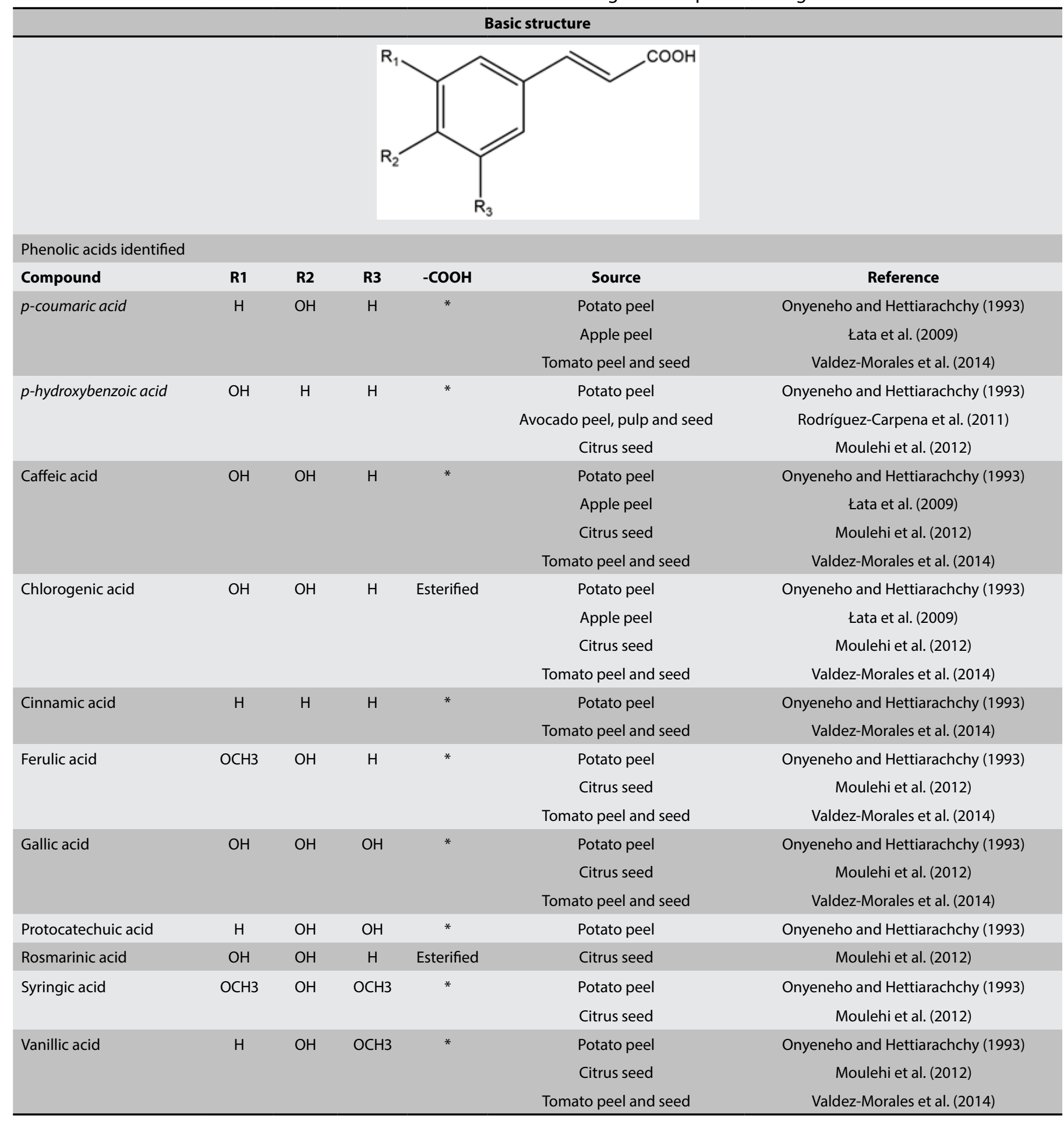


Table 2. Basic structure of flavonoids identified in some agro-industrial by-products.

Tabla 2. Estructura básica de algunos flavonoides identificados en subproductos agroindustriales.

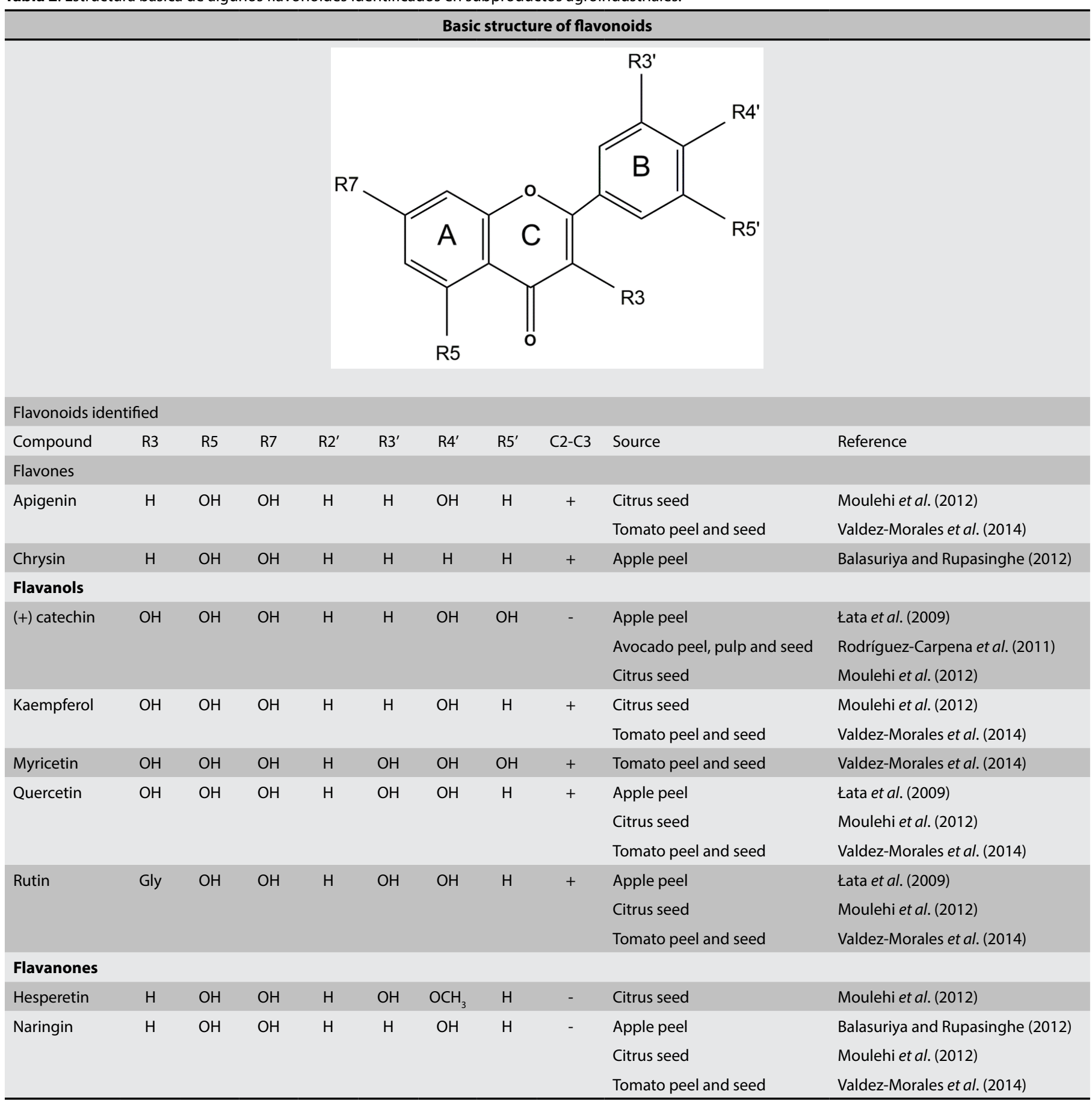

(+) double; (-) single.

fungal mycelia is widely produced in SSF using substrates such as grains, sawdust or wood from different plant species (Yang and Liau, 1998). Nevertheless, it has been reported that SCF improves potential advantage than SSF, because inoculums can be uniformly dispersed in the substrate, and the time and/or harvest speed are reduced (Yang and Liau, 1998; Xu and Zhu, 2011; Xu et al., 2015).

Moreover, fungal mycelia production during the fermentation process varies extensively, depending on the species of fungus and environmental or culture conditions used (temperature, initial $\mathrm{pH}$, surface-aeration, aeration rate, rotating speed, and stimulatory agents, among others), which consequently affect phytochemical extraction from the substrate (Yang and Liau, 1998; Xu and Zhu, 2011; Xu et al., 2015).

Phenolic Compounds Extraction by Fungal Fermentation In relation to the aforementioned, the use of agro-in- 
dustrial by-products as substrates in combination with fungal fermentation-assisted extraction (SSF and SCF), are considered an alternative method for the extraction of phytochemicals, including triterpenoids, polysaccharides, and phenolic compounds, which could be obtained through agro-industrial by-products (Xu and Zhu, 2011; Xu et al., 2014; Xu et al., 2015; Dey et al., 2016).

In this context, the extraction of phenolic compounds and triterpenoids from citrus peel like pomelo, lemon, orange, and tangerine, through SCF $\left(25^{\circ} \mathrm{C}\right.$ at $100 \mathrm{rpm}, 28$ d) with Antrodia cinnamomea has been reported (Ma et al., 2014). Also, polysaccharide and triterpenoid extraction from citrus peels, including pomelo, lemon, orange, and grapefruit, using SCF $\left(25^{\circ} \mathrm{C}\right.$ at $\left.100 \mathrm{rpm}, 28 \mathrm{~d}\right)$ with $A$. cinnamomea was also demonstrated (Yang et al., 2012). Xu and Zhu (2011), reported the extraction of phenolic compounds with antioxidant properties (DPPH and hydroxyl scavenging activity) from ground corn stover by SCF $\left(28^{\circ} \mathrm{C}\right.$ at $\left.150 \mathrm{rpm}, 12 \mathrm{~d}\right)$ using Inonotus obliquus. In addition, Vattem and Shetty (2002) demonstrated the extraction of phenolic compounds such as ellagic acid, resveratrol and rosmarinic acid with antioxidant properties (antiradical DPPH and $\beta$-carotene antioxidant protection factor) from cranberry pomace by SSF $\left(28^{\circ} \mathrm{C}, 16\right.$ d) using Rhizopus oligosporus.

Additionally, table 3 compile literature reports focused on the extraction of phenolic compounds with antioxidant and antibacterial properties, from agro-industrial by-products using fungal fermentation-assisted extraction

Table 3. Obtaining phenolic compounds from agro-industrial by-products through fungal fermentation-assisted extraction.

Tabla 3. Obtención de compuestos fenólicos de subproductos agroindustriales mediante extracción-asistida por fermentación fúngica.

\begin{tabular}{|c|c|c|c|c|}
\hline Substrate & Fungi & Fermentation & Relevant results & Reference \\
\hline Black rice bran & $\begin{array}{c}\text { Aspergillus awam- } \\
\text { ori and Aspergillus } \\
\text { oryzae }\end{array}$ & SSF & $\begin{array}{l}\text { 'Fungal fermentation effect on phenolic compounds' } \\
\nabla \text { Total phenolic and anthocyanin content, in the order A. awamori }>A \text {. oryzae } \\
\text { Total phenolic content obtained by decomposing anthocyanin content } \\
\Delta \text { Protocatechuic, OH-benzoic, vanillic, caffeic, } p \text {-coumaric and ferulic acids } \\
\text { 'Fungal fermentation effect on bioactivity' } \\
\Delta \text { DPPH' radical-scavenging activity }\end{array}$ & $\begin{array}{l}\text { Shin et al. } \\
(2019)\end{array}$ \\
\hline Peanut press cake & $\begin{array}{c}\text { Aspergillus } \\
\text { awamori }\end{array}$ & SSF & $\begin{array}{l}\text { 'Fungal fermentation effect on phenolic compounds' } \\
\Delta \text { Total phenolic, flavonoid and tannin content } \\
\text { 'Fungal fermentation effect on bioactivity' } \\
\text { A ABTS*+ and DPPH' radical-scavenging activity } \\
\Delta \text { Metal chelating activity }\end{array}$ & $\begin{array}{c}\text { Sadh et al. } \\
(2018)\end{array}$ \\
\hline Rice bran extract & $\begin{array}{c}\text { Aspergillus oryzae } \\
\text { and Rhizopus } \\
\text { oryzae }\end{array}$ & SSF & $\begin{array}{l}\text { 'Fungal fermentation effect on phenolic compounds' } \\
\text { A Ferulic, caffeic, and protocatechuic acids, by } A \text {. oryzae } \\
\text { A Sinapic, vanillic, caffeic, syringic, protocatechuic, and 4-hydroxybenzoic } \\
\text { acids, by } R \text {. oryzae } \\
\text { 'Fungal fermentation effect on bioactivity' } \\
\text { - FRAP, by both fungi } \\
\text { - DPPH' radical-scavenging activity }\end{array}$ & $\begin{array}{l}\text { Razak et al. } \\
\text { (2017) }\end{array}$ \\
\hline $\begin{array}{l}\text { Corncob, pea } \\
\text { pod, rice straw, } \\
\text { sugarcane ba- } \\
\text { gasse, and wheat } \\
\text { straw }\end{array}$ & $\begin{array}{l}\text { Aspergillus terreus } \\
\text { and Penicillium } \\
\text { citrinum }\end{array}$ & SSF & $\begin{array}{l}\text { 'Fungal fermentation effect on phenolic compounds' } \\
\Delta \text { Total phenolic content, by both fungi } \\
\text { 'Fungal fermentation effect on bioactivity' } \\
\text { A DPPH' and NO' radical-scavenging activity, by both fungi } \\
\text { A Fe }{ }^{2+} \text { scavenging activity, by both fungi } \\
\Delta \text { FRAP, by both fungi }\end{array}$ & $\begin{array}{l}\text { Chandra and } \\
\text { Arora (2016) }\end{array}$ \\
\hline Plum fruit & $\begin{array}{l}\text { Aspergillus niger } \\
\text { and Rhizopus } \\
\text { oligosporus }\end{array}$ & SSF & 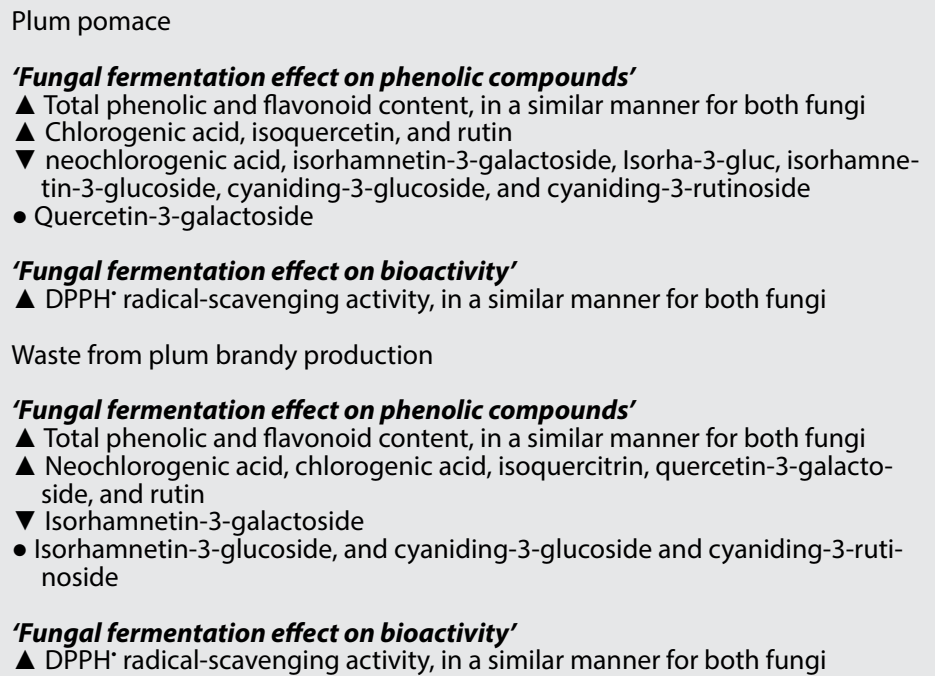 & $\begin{array}{l}\text { Dulf et al. } \\
(2016)\end{array}$ \\
\hline
\end{tabular}


Vargas-Sánchez et al: Extraction of phenolic compounds from agro-industrial / XXIII (3): 66-77 (2021)

\begin{tabular}{|c|c|c|c|c|}
\hline Substrate & Fungi & Fermentation & Relevant results & Reference \\
\hline Apple pomace & Rhizopus oryzae & SSF and SCF & $\begin{array}{l}\text { 'Fungal fermentation effect on phenolic compounds' } \\
\Delta \text { Fumaric acid production, by both culture methods }\end{array}$ & $\begin{array}{l}\text { Das et al. } \\
(2015)\end{array}$ \\
\hline \multirow[t]{2}{*}{ Orchid } & \multirow[t]{2}{*}{$\begin{array}{l}\text { Fusarium avenace- } \\
\text { um and Fusarium } \\
\text { oxysporum }\end{array}$} & \multirow[t]{2}{*}{ SSF } & $\begin{array}{l}\text { 'Fungal fermentation effect on phenolic compounds' } \\
\Delta \text { Total phenolic content }\end{array}$ & \multirow[t]{2}{*}{$\begin{array}{l}\text { Dong et al. } \\
\text { (2015) }\end{array}$} \\
\hline & & & $\begin{array}{l}\text { 'Fungal fermentation effect on bioactivity' } \\
\text { A DPPH' and ABTS'+ radical-scavenging activity, as well as reducing power } \\
\Delta \text { Inhibition of Staphylococcus aureus and Bacillus subtilis growth }\end{array}$ & \\
\hline \multirow[t]{2}{*}{ Peanut shell } & \multirow[t]{2}{*}{ Inonotus obliquus } & \multirow[t]{2}{*}{ SCF } & $\begin{array}{l}\text { 'Fungal fermentation effect on phenolic compounds' } \\
\text { A Phenolic compounds such as epigallocatechin-3-gallate, epicatechin-3-gallate, } \\
\text { phelligridin G, davallialactone, and inoscavin B } \\
\nabla \text { Phenolic acid, including gallic and ferulic acids }\end{array}$ & \multirow[t]{2}{*}{$\begin{array}{l}\text { Xu et al. } \\
\text { (2014) }\end{array}$} \\
\hline & & & $\begin{array}{l}\text { 'Fungal fermentation effect on bioactivity' } \\
\triangle \mathrm{DPPH} \text { ' and 'OH radical-scavenging activity }\end{array}$ & \\
\hline Algae & Candida utilis & SCF & $\begin{array}{l}\text { 'Fungal fermentation effect on bioactivity' } \\
\Delta \text { Inhibition of methicillin-resistant Staphylococcus aureus }\end{array}$ & $\begin{array}{l}\text { Eom et al. } \\
(2013)\end{array}$ \\
\hline \multirow[t]{3}{*}{ Herbal residues } & \multirow[t]{3}{*}{ Aspergillus oryzae } & \multirow[t]{3}{*}{ SSF } & $\begin{array}{l}\text { 'Fungal fermentation effect on phenolic compounds' } \\
\Delta \text { Gallic acid formation }\end{array}$ & \multirow[t]{3}{*}{$\begin{array}{l}\text { Wen et al. } \\
\text { (2013) }\end{array}$} \\
\hline & & & $\begin{array}{l}\text { 'Fungal fermentation effect on bioactivity' } \\
\Delta \text { DPPH' radical-scavenging activity and reducing power }\end{array}$ & \\
\hline & & & $\begin{array}{l}\text { Anhibition of Bacillus cereus, Listeria monocytogenes, Staphylococcus aureus, and } \\
\text { Escherichia coli }\end{array}$ & \\
\hline \multirow[t]{2}{*}{$\begin{array}{l}\text { Sugarcane ba- } \\
\text { gasse }\end{array}$} & \multirow[t]{2}{*}{ Inonotus obliquus } & \multirow[t]{2}{*}{ SCF } & $\begin{array}{l}\text { 'Fungal fermentation effect on phenolic compounds' } \\
\text { A Phenolic compounds such as epicatechin-3-gallate, epigallocatechin-3-gallate, } \\
\text { and phelligridin G }\end{array}$ & \multirow[t]{2}{*}{$\begin{array}{l}\text { Zhu and Xu } \\
(2013)\end{array}$} \\
\hline & & & $\begin{array}{l}\text { 'Fungal fermentation effect on bioactivity' } \\
\triangle \mathrm{DPPH} \text { and 'OH radical-scavenging activity }\end{array}$ & \\
\hline $\begin{array}{l}\text { Coffee silver- } \\
\text { skin and coffee } \\
\text { grounds }\end{array}$ & $\begin{array}{l}\text { Aspergillus ustus, } \\
\text { Aspergillus niger, } \\
\text { Neurospora crassa, } \\
\text { and Penicillium } \\
\text { purpurogenum }\end{array}$ & SSF & $\begin{array}{l}\text { 'Fungal fermentation effect on phenolic compounds' } \\
\Delta \text { Total phenolic content }\end{array}$ & $\begin{array}{l}\text { Machado et } \\
\text { al. (2012) }\end{array}$ \\
\hline \multirow[t]{2}{*}{$\begin{array}{l}\text { Pineapple and } \\
\text { guava }\end{array}$} & \multirow[t]{2}{*}{$\begin{array}{l}\text { Rhizopus oligos- } \\
\quad \text { porus }\end{array}$} & \multirow[t]{2}{*}{ SSF } & $\begin{array}{l}\text { 'Fungal fermentation effect on phenolic compounds' } \\
\Delta \text { Total phenolic content }\end{array}$ & \multirow[t]{2}{*}{$\begin{array}{l}\text { Sousa and } \\
\text { Correia (2012) }\end{array}$} \\
\hline & & & $\begin{array}{l}\text { 'Fungal fermentation effect on bioactivity' } \\
\nabla \text { DPPH' radical-scavenging activity }\end{array}$ & \\
\hline Corn cob & Yarrowia lipolytica & SCF & $\begin{array}{l}\text { 'Fungal fermentation effect on phenolic compounds' } \\
\Delta \text { Ferulic acid production }\end{array}$ & $\begin{array}{l}\text { Huang et al. } \\
\quad(2011)\end{array}$ \\
\hline Cashew husk & Aspergillus oryzae & SSF & $\begin{array}{l}\text { 'Fungal fermentation effect on phenolic compounds' } \\
\Delta \text { Gallic acid production }\end{array}$ & $\begin{array}{l}\text { Lokeshwari } \\
\text { and Reddy } \\
\quad(2010)\end{array}$ \\
\hline Citrus peel & Cordyceps sinensis & SCF & $\begin{array}{l}\Delta \text { Total phenolic and flavonoids content } \\
\Delta \text { ABTS }^{++} \text {radical-scavenging activity }\end{array}$ & $\begin{array}{l}\text { Choi et al. } \\
(2010)\end{array}$ \\
\hline Wheat bran & $\begin{array}{l}\text { Agrocybe chaxin- } \\
\text { gu, Auricularia } \\
\text { auricula-judae, } \\
\text { Cordyceps militaris, } \\
\text { Hericium erina- } \\
\text { ceus, and Pleurotus } \\
\text { ostreatus }\end{array}$ & SCF & $\begin{array}{l}\text { 'Fungal fermentation effect on phenolic compounds' } \\
\Delta \text { Ferulic acid production, in the order H. erinaceus }>\text { P. ostreatus }>\text { C. militaris }\end{array}$ & Xie et al. 2010 \\
\hline $\begin{array}{l}\text { Valonia acorns } \\
\text { extract }\end{array}$ & $\begin{array}{l}\text { Aspergillus oryzae } \\
\text { and Trichoderma } \\
\text { reesei }\end{array}$ & SCF & $\begin{array}{l}\text { 'Fungal fermentation effect on phenolic compounds' } \\
\Delta \text { Ellagic acid production, in the order } A \text {. oryzae }>\text { T. reesei }\end{array}$ & $\begin{array}{l}\text { Huang et al. } \\
\text { (2007) }\end{array}$ \\
\hline $\begin{array}{l}\text { Shrimp and crab } \\
\text { shell powder }\end{array}$ & $\begin{array}{l}\text { Monascus purpu- } \\
\text { reus }\end{array}$ & SCF & $\begin{array}{l}\text { 'Fungal fermentation effect on bioactivity' } \\
\text { Antimicrobial effect against Bacillus subtilis, Bacillus cereus, Pseudomonas aeru- } \\
\text { ginosa, Staphylococcus aureus and Escherichia coli }\end{array}$ & $\begin{array}{l}\text { Wang et al. } \\
\text { (2002) }\end{array}$ \\
\hline Tannic acid & $\begin{array}{l}\text { Aspergillus } \\
\text { awamori }\end{array}$ & SCF & $\begin{array}{l}\text { 'Fungal fermentation effect on phenolic compounds' } \\
\Delta \text { Gallic acid production }\end{array}$ & $\begin{array}{l}\text { Seth and } \\
\text { Chand (2000) }\end{array}$ \\
\hline
\end{tabular}

$(\mathbf{\Lambda})$, significant increase with respect to the control group; $(\boldsymbol{\nabla})$, significant reduction with respect to the control group; $(\bullet)$, without significant differences with respect to the control group. 
(SSF and SCF). Mycelial growth during fungal fermentation depends on the nutrient supply (nitrogen, phosphorus and carbon) and any type of energy source or substrate, as well as substrate digestibility, which are essential for extraction of bioactive secondary metabolites (Hölker et al., 2004).

Likewise, an increase in enzyme production (lipases, xylanase, pectinase, proteases, cellulolytic, and ligninolytic enzymes) during fungal fermentation has been demonstrated (Hölker et al., 2004; Sadh et al., 2018). The enzymatic hydrolysis produced during fungal fermentation increase the extraction of phenolic compounds, such as $p$-coumaric, caffeic, chlorogenic, ferulic, protocatechuic, sinapic, syringic, and vanillic acids, quercetin, and rutin. Also, improve antioxidant activity like antiradical (DPPH ${ }^{\circ}$ and $\mathrm{ABTS}^{-+}$activity), chelating metal properties, ferric reducing antioxidant power, and nitric oxide chelating properties. As well as antimicrobial activity by microbial growth and foodborne pathogens reduction (Hölker et al., 2004; Das et al., 2015; Dong et al., 2015; Dulf et al., 2016; Razak et al., 2017; Sadh et al., 2018; Shin et al., 2019). Thus, the enzymatic hydrolysis produced during fungal fermentation appears to be an attractive strategy to extract phenolic compounds with potential uses as food additives (Papaspyridi et al., 2012).

\section{Phenolic Compounds as Possible Meat and Meat Product Additives}

The NOM-213-SSA1-2002 define a 'food additive' as 'those substances, which added directly to food and beverages during their elaboration, provide or intensify aroma, color, and flavor, to improve stability and conservation'. Also, the FDA (2008) indicate that a 'Food Additive' is 'any substance that when use directly or indirectly, become a component or otherwise affect the characteristics of any food, including any substance intended for use in packaging, production, manufacturing, processing, preparation, treatment, transportation or storage of food; and including any source of radiation intended for such use'. The Codex Alimentarius (2017) defined it as 'any substance that, regardless of its nutritional value, is intentionally added to a food in controlled quantities for technological purposes'.

Moreover, in the meat and meat products industry, additives are widely employed for preservative purposes (i.e., as antioxidants and antimicrobials). An antioxidant additive, is defined as a'substance added to foods to prevent the oxygen present in the air from causing undesirable changes in flavor and color' (USDA, 2015). In another context, an antimicrobial additive, is defined as 'a substance that meets the definition of food additive and is used to control microorganisms such as bacteria, viruses, fungi, among others, in food or food contact items' (FDA, 2008).

The following is a list of additives commonly used in meat and meat products as preservatives are: a-tocopherol (E307), acetic acid (E260), ascorbic acid (E300), citric acid (E330), erythorbic acid (E315), fumaric acid (E297), lactic acid (E270), sorbic acid (E200), tartaric acid (E334), sodium ascorbate (E301), calcium ascorbate (E302), sodium benzoate (E211), butylhydroxyanisole (E320), and butylhydroxytoluene (E321). Also, calcium carbonate (E170i), sodium citrate (E330), potassium citrate (E332), tricalcium citrate (E333iii), trisodium citrate (E331iii), isopropyl citrate (E384), sodium diacetate (E262ii), sodium erythorbate (E316), ethyl lauroyl arginate (E243), propyl gallate (E310), nitrite/sodium nitrate (E250 and E252), tert-butylhydroquinone (E319), potassium sorbate (E202), among others (NOM-122-SSA1-1994; FDA, 2004; European Commission, 2014; FAO, 2018). The preservative compounds mentioned above have phenolic groups in their structure, which in phenolic compounds (phenolic acid and flavonoids) are widely associated with their antioxidant and antimicrobial activity (Sova, 2012).

Moreover, extensive research has demonstrated that lipid oxidation and microbial growth, are the main factors involved in the quality loss of raw and cooked meat products. These factors lead to the formation of some compounds that affect sensory attributes, including changes in texture, odor, flavor, and color, which consequently have an adverse effect on meat acceptability and meat purchase intention (Faustmant et al., 2010; Aziz and Karboune, 2018). Therefore, there have been efforts to obtain antioxidant and antimicrobial compounds from natural sources, including agro-industrial by-products (Faustmant et al., 2010; Jiang and Xiong, 2016).

Table 4 shows the possible uses of phenolic compounds, obtained by SSF and SCF using agro-industrial residues as substrate, including as meat and meat product additives. In this context, it has been demonstrated that phenolic compounds and flavonoids can preserve raw and cooked meat and meat products from different species (beef, camel, chicken, and pork), against undesirable changes caused by lipid oxidation and microbial growth during refrigerated

Table 4. Uses of phenolic compounds as additives for meat and meat products.

Tabla 4. Usos de compuestos fenólicos como aditivos para carne y productos cárnicos.

\begin{tabular}{|c|c|c|c|}
\hline \multicolumn{4}{|c|}{ As an antioxidant additive } \\
\hline Phenolic compounds & Conditions & Relevant results & References \\
\hline $\begin{array}{l}\text { Flavonoids: catechin } \\
\text { Phenolic acids: tannic, caffeic, } \\
\text { and gallic }\end{array}$ & $\begin{array}{l}\text { Product: Minced camel } \\
\text { meat } \\
\text { Storage: } 4^{\circ} \mathrm{C} \text { for } 9 \text { days } \\
\text { Addition level: } 200 \mathrm{ppm}\end{array}$ & $\begin{array}{l}\text { Inhibition of lipid oxidation (catechin } 72.7 \% \text {, as well as tannic } 95.5 \% \text {, } \\
\text { caffeic } 80 \% \text {, and gallic acids } 70 \% \text { approximately) } \\
\text { Red color, } 1 \text { point in sensory score for all phenolic compounds }\end{array}$ & Maqsoo et al. (2015) \\
\hline $\begin{array}{l}\text { Phenolic acids: caffeic, } t \text {-cin- } \\
\text { namic, } p \text {-coumaric, ferulic, gallic, } \\
p \text {-hydroxybenzoic, gentisic, } \\
\text { sinapic, and syringic }\end{array}$ & $\begin{array}{l}\text { Product: Beef } \\
\text { Storage: } 4^{\circ} \mathrm{C} \text { for } 6 \text { days } \\
\text { Addition level: } 0.05 \\
\mathrm{mmol} / \mathrm{kg}\end{array}$ & $\begin{array}{l}\Delta \text { Inhibition of lipid oxidation precooked beef in the order sinapic acid } \\
>\text { caffeic acid }>\text { ferulic acid }>\text { gentisic acid }>\text { syringic acid }>t \text {-cinnamic } \\
\text { acid }>p \text {-coumaric acid }>p \text {-hydroxybenzoic acid }\end{array}$ & $\begin{array}{l}\text { Brettonnet et al. } \\
\quad(2010)\end{array}$ \\
\hline
\end{tabular}




\begin{tabular}{|c|c|c|c|}
\hline Phenolic compounds & Conditions & Relevant results & References \\
\hline Flavonoids: quercetin and rutin & $\begin{array}{l}\text { Product: beef patties } \\
\text { Storage: } 2^{\circ} \mathrm{C} \text { for } 11 \text { days } \\
\text { Addition level: } 1 \text { and } \\
5 \mathrm{mM}\end{array}$ & $\begin{array}{l}\boldsymbol{\nabla} \text { L* values in concentration dependence (quercetin } 1.9 \% \text {; rutin } 3.0 \% \text { ) } \\
\nabla \text { C values in concentration dependence (quercetin } 12.3 \% \text {; rutin } 16.6 \% \text { ) } \\
\Delta \text { h values in concentration dependence (quercetin } 8.9 \% \text {; rutin } 16.4 \% \text { ) } \\
\Delta \text { Inhibition of metmyoglobin formation in concentration dependence } \\
\text { (quercetin } 47.0 \% \text { approximately; rutin } 66.0 \% \text { approximately) } \\
\Delta \text { Inhibition of lipid oxidation in concentration dependence (quercetin } \\
14.3 \% \text { ) } \\
\nabla \text { Inhibition of lipid oxidation (rutin }-23.8 \% \text { ) }\end{array}$ & Bekhit et al. (2004) \\
\hline Flavonoids: quercetin & $\begin{array}{l}\text { Product: cook-chill } \\
\text { chicken } \\
\text { Storage: } 5^{\circ} \mathrm{C} \text { for } 5 \text { days } \\
\text { Addition level: } 1.6 \% \text { and } \\
3.0 \%\end{array}$ & $\begin{array}{l}\text { Inhibition of lipid oxidation (83.9\% and } 97.3 \% \text { in concentration } \\
\text { dependence) }\end{array}$ & $\begin{array}{l}\text { Karastogiannidou } \\
\text { (1999) }\end{array}$ \\
\hline $\begin{array}{l}\text { Flavonoids: (-)-epicatechin } \\
\text { (EC), (-)-epigallocatechin (EGC), } \\
\text { (-)-epicatechin gallate (ECG), } \\
\text { and (-)-epigallocatechin gallate } \\
\text { (EGCG) }\end{array}$ & $\begin{array}{l}\text { Product: ground white } \\
\text { muscle of mackerel } \\
\text { Storage: cooked at } 75 \\
{ }^{\circ} \mathrm{C}, 4^{\circ} \mathrm{C} \text { for } 7 \text { days } \\
\text { Addition level: EC and } \\
\text { EGC ( } 200 \mathrm{mg} / \mathrm{kg}) . \mathrm{ECG} \\
\text { and EGCG }(300 \mathrm{mg} / \mathrm{kg})\end{array}$ & $\Delta$ Inhibition of lipid oxidation (EC 40.5\%; EGC, ECG, and EGCG 65.5\%) & $\begin{array}{l}\text { He and Shahidi } \\
(1997)\end{array}$ \\
\hline $\begin{array}{l}\text { Flavonoids: kaempferol, morin, } \\
\text { myricetin, naringenin, naringin, } \\
\text { quercetin, and rutin } \\
\text { Phenolic acids: gallic, caffeic, } \\
\text { coumaric, ferulic, syringic, } \\
\text { vanillic, sinapic, chlorogenic, } \\
\text { and tannic }\end{array}$ & $\begin{array}{l}\text { Product: cooked ground } \\
\text { pork } \\
\text { Storage: } 4^{\circ} \mathrm{C} \text { for } 4 \text { weeks } \\
\text { Addition level: } 30 \text { and } \\
200 \text { ppm }\end{array}$ & $\begin{array}{l}\text { Inhibition of lipid oxidation in concentration dependence } \\
\text { Kaempferol (95.3\%), morin (96.4\%), myricetin }(98.7 \%) \text {, naringenin } \\
\text { (3.3\%), naringin }(2.3 \%) \text {, quercetin }(98.9 \%) \text {, rutin }(33.0 \%) \text {, as well as } \\
\text { gallic }(73.5 \%) \text {, caffeic }(69.3 \%) \text {, coumaric }(54.3 \%) \text {, ferulic }(56.6 \%) \text {, syrin- } \\
\text { gic }(55.3 \%) \text {, vanillic }(23.6 \%) \text {, sinapic }(38.5 \%) \text {, chlorogenic }(35.0 \%) \text {, and } \\
\text { tannic }(98.9 \%) \text { acids }\end{array}$ & Shahidi et al. (1993) \\
\hline $\begin{array}{l}\text { Flavonoids: kaempferol, morin, } \\
\text { myricetin, naringenin, naringin, } \\
\text { quercetin, and rutin } \\
\text { Phenolic acids: ellagic, gallic, } \\
\text { vanillic, syringic, and tannic }\end{array}$ & $\begin{array}{l}\text { Product: pork model } \\
\text { system, cooked at } 75^{\circ} \mathrm{C} \\
\text { Storage: } 4^{\circ} \mathrm{C} \text { for } 3 \text { weeks } \\
\text { Addition level: } 200 \text { ppm }\end{array}$ & 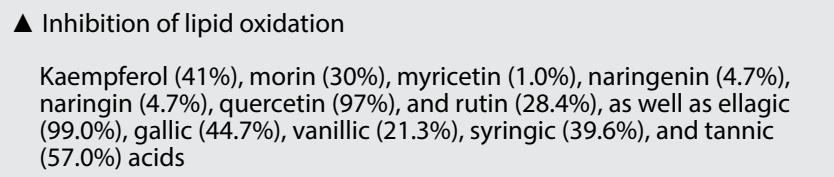 & Shahidi et al. (1992) \\
\hline \multicolumn{4}{|l|}{ As an antimicrobial additive } \\
\hline Phenolic compounds & Conditions & Relevant results & References \\
\hline $\begin{array}{l}\text { Flavonoids: catechin } \\
\text { Phenolic acids: tannic, caffeic, } \\
\text { and gallic }\end{array}$ & $\begin{array}{l}\text { Product: Minced camel } \\
\text { meat } \\
\text { Storage: } 4{ }^{\circ} \mathrm{C} \text { for } 9 \text { days } \\
\text { Addition level: } 200 \text { ppm }\end{array}$ & $\begin{array}{l}\Delta \text { Inhibition of mesophilic bacteria count, } 1 \text { log approximately (catechin } \\
\text { and tannic acids) } \\
\text { Inhibition of psychrotrophic bacteria count, } 1 \text { log approximately (cate- } \\
\text { chin, tannic, and gallic acids) }\end{array}$ & Maqsoo et al. (2015) \\
\hline $\begin{array}{l}\text { Flavonoids: rutin } \\
\text { Phenolic acids: caffeic acid and } \\
\text { p-coumaric }\end{array}$ & $\begin{array}{l}\text { Product: chicken soup } \\
\text { Storage: } 4 \text { and } 25^{\circ} \mathrm{C} \\
\text { for } 48 \mathrm{~h} \\
\text { Addition level: } 0.2 \mathrm{mg} / \\
\mathrm{mL}\end{array}$ & $\begin{array}{l}\text { Inhibition of Staphylococcus aureus growth (100\% by all phenolic } \\
\text { compounds) }\end{array}$ & $\begin{array}{l}\text { Stojković et al. } \\
\text { (2013) }\end{array}$ \\
\hline Phenolic acids: benzoic & $\begin{array}{l}\text { Product: Raw and } \\
\text { cooked chicken meat } \\
\text { Storage: } 4 \text { and } 20^{\circ} \mathrm{C} \text { for } \\
14 \text { days } \\
\text { Addition level: } 5000 \\
\text { ppm }\end{array}$ & $\begin{array}{l}\Delta \text { Inhibition of Listeria monocytogenes and growth in raw and cooked } \\
\text { meat ( } 1.2 \text { and } 3.5 \text { log, respectively) } \\
\Delta \text { Inhibition of Salmonella typhimurium and growth in raw and cooked } \\
\text { meat ( } 1.2 \text { log by both) }\end{array}$ & $\begin{array}{l}\text { Ravichandran et al. } \\
\text { (2011) }\end{array}$ \\
\hline $\begin{array}{l}\text { Flavonoids: Mixture of querce- } \\
\text { tin and rutin } \\
\text { Phenolic acids: } \\
\text { Mixture of gallic and caffeic } \\
\text { Mixture of gallic and protocat- } \\
\text { echuic }\end{array}$ & $\begin{array}{l}\text { Product: meat model } \\
\text { system } \\
\text { Storage: } 4{ }^{\circ} \mathrm{C} \text { for } 24 \mathrm{~h} \\
\text { days } \\
\text { Addition level: } 100 \text { and } \\
200 \mathrm{mg}\end{array}$ & $\begin{array}{l}\text { Inhibition of Listeria monocytogenes growth in concentration depen- } \\
\text { dence (mixture quercetin and rutin } 6.7 \mathrm{log} \text {; mixture gallic and caffeic } \\
\text { acids } 6.3 \text { log; mixture gallic and protocatechuic acids } 3.7 \mathrm{log} \text { ) }\end{array}$ & $\begin{array}{l}\text { Rodríguez-Vaquero } \\
\text { et al. (2011) }\end{array}$ \\
\hline $\begin{array}{l}\text { Phenolic acids: carvacrol and } \\
\text { thymol }\end{array}$ & $\begin{array}{l}\text { Product: bovine meat } \\
\text { stake } \\
\text { Storage: } 7^{\circ} \mathrm{C} \text { for } 96 \mathrm{~h} \\
\text { Addition level: }<1 \mu \mathrm{L} / \mathrm{mL}\end{array}$ & $\begin{array}{l}\text { Inhibition of Staphylococcus aureus growth of carvacrol and thymol in } \\
\text { combination with organic acids (lactic and acetic) }\end{array}$ & $\begin{array}{c}\text { De Oliviera et al. } \\
(2010)\end{array}$ \\
\hline $\begin{array}{l}\text { Flavonoids: Mixture of querce- } \\
\text { tin and rutin } \\
\text { Phenolic acids: } \\
\text { Mixture of gallic and caffeic } \\
\text { Mixture of gallic and protocat- } \\
\text { echuic }\end{array}$ & $\begin{array}{l}\text { Product: meat model } \\
\text { system } \\
\text { Storage: } 20^{\circ} \mathrm{C} \text { for } 14 \\
\text { days } \\
\text { Addition level: } 100 \text { and } \\
200 \mathrm{mg}\end{array}$ & $\begin{array}{l}\text { Concentration- and temperature-dependent inhibition of Escherichia } \\
\text { coli growth (mixture of quercetin and rutin } 100 \% \text {; mixture of gallic and } \\
\text { caffeic acids } 100 \% \text {; mixture of gallic and protocatechuic acids } 50 \% \\
\text { approximately) }\end{array}$ & $\begin{array}{l}\text { Rodríguez-Vaquero } \\
\text { et al. (2010) }\end{array}$ \\
\hline
\end{tabular}

$(\boldsymbol{\Lambda})$, significant increase with respect to the control group; $(\boldsymbol{\nabla})$, significant reduction with respect to the control group. 
storage (Stojković et al., 2013; Maqsoo et al., 2015). Furthermore, phenolic compounds can act through two pathways: (1) by breaking chain reactions triggered by free radicals, which implies hydrogen atom transfer (HAT), then electron transfer followed by a proton transfer mechanism (SET-PT) and sequential proton-loss electron-transfer (SPLET), and (2) by reducing metals such as copper $\left(\mathrm{Cu}^{2+}\right)$ and iron $\left(\mathrm{Fe}^{3+}\right)$ (Marković et al., 2012). Additionally, phenolic compounds can act against nucleic acid and protein synthesis and alter the components of cellular membranes (Cushnie and Lamb, 2005).

\section{CONCLUSION}

The agro-industrial by-products are an important source of phenolic compounds, including phenolic acids and flavonoids. The uses of agro-industrial residues as substrates (seeds, pulps, and peels) during fungal fermentation-assisted extraction (SSF and SCF), can be used as an alternative or complementary strategy to obtain phenolic compounds like rustic, conventional and unconventional extraction methods. These compounds could be use as antioxidant and antimicrobial additives to extend the shelf life of raw and cooked meat and meat products from different species (beef, camel, chicken, and pork) during refrigerated storage.

\section{ACKNOWLEDGEMENTS}

The authors gratefully acknowledge Cátedras CONACYT for the fellowship of the project (\#739).

\section{REFERENCES}

Aziz, M. and Karboune, S. 2018. Natural antimicrobial/antioxidant agents in meat and poultry products as well as fruits and vegetables: a review. Critical Reviews in Food Science and Nutrition. 58: 486-511.

Azmir, J., Zaidul, I.S.M., Rahman, M.M., Sharif, K.M., Mohamed, A., Sahena, F., Jahurul, M.H.A. and Omar, A.K.M. 2013. Techniques for extraction of bioactive compounds from plant materials: A review. Journal of Food Engineering. 117: 426-436.

Balasuriya, N. and Rupasinghe, H.V. 2012. Antihypertensive properties of flavonoid-rich apple peel extract. Food Chemistry. 135: 2320-2325.

Bekhit, A.E.D., Geesink, G.H., Ilian, M.A., Morton, J.D., Sedcole, J.R. and Bickerstaffe, R. 2004. Pro-oxidant activities of carnosine, rutin and quercetin in a beef model system and their effects on the metmyoglobin-reducing activity. European Food Research and Technology. 218: 507-514.

Brettonnet, A., Hewavitarana, A., DeJong, S. and Lanari, M.C. 2010. Phenolic acids composition and antioxidant activity of canola extracts in cooked beef, chicken, and pork. Food Chemistry. 121: 927-933.

Castañeda-Casasola, C., Arana-Cuenca, A., Favela-Torres, E., Anducho-Reyes, M.A., González, A.E. and Téllez-Jurado, A. 2018. Xylanase enzymes production by Aspergillus fumigatus in solid state fermentation and submerge fermentation. Revista Mexicana de Ingeniería Química. 17: 47-61.
Chandra, P. and Arora, D.S. 2016. Production of antioxidant bioactive phenolic compounds by solid-state fermentation on agro-residues using various fungi isolated from soil. Asian Journal of Biotechnology. 8: 8-15.

Choi, J.W., Ra, K.S., Kim, S.Y., Yoon, T.J., Yu, K.W., Shin, K.S., Lee, S.P. and Suh, H.J. 2010. Enhancement of anti-complementary and radical scavenging activities in the submerged culture of Cordyceps sinensis by addition of citrus peel. Bioresource Technology. 101: 6028-6034.

Comisión del Codex Alimentarius, Normas internacionales de los alimentos; Norma general para los aditivos alimentarios [Accesses 2 march 2020] 2017. Available in: http://www. fao.org/gsfaonline/docs/CXS_192s.pdf. (Fecha de consulta, Septiembre de 2019).

Cushnie, T.P.T. and Lamb, A.J. 2005. Antimicrobial activity of flavonoids. International Journal of Antimicrobial Agents. 26: 343-356.

Das, R.K., Brar, S.K. and Verma, M. 2015. A fermentative approach towards optimizing directed biosynthesis of fumaric acid by Rhizopus oryzae 1526 utilizing apple industry waste biomass. Fungal Biology. 119: 1279-1290.

De Oliveira, C.E.V., Stamford, T.L.M., Neto, N.J.G. and de Souza, E.L. 2010. Inhibition of Staphylococcus aureus in broth and meat broth using synergies of phenolics and organic acids. International Journal of Food Microbiology. 137: 312-316.

Dey, T.B., Chakraborty, S., Jain, K.K., Sharma, A. and Kuhad, R.C. 2016. Antioxidant phenolics and their microbial production by submerged and solid-state fermentation process: A review. Trends in Food Science \& Technology. 53: 60-74.

Dong, J.W., Cai, L., Xiong, J., Chen, X.H., Wang, W.Y., Shen, N., Liu, B.L. and Ding, Z.T. 2015. Improving the antioxidant and antibacterial activities of fermented Bletilla striata with Fusarium avenaceum and Fusarium oxysporum. Process Biochemistry. 50: 8-13.

Dulf, F.V., Vodnar, D.C. and Socaciu, C. 2016. Effects of solidstate fermentation with two filamentous fungi on the total phenolic contents, flavonoids, antioxidant activities and lipid fractions of plum fruit (Prunus domestica L.) byproducts. Food Chemistry. 209: 27-36.

Eom, S.H., Lee, D.S., Kang, Y.M., Son, K.T., Jeon, Y.J. and Kim, Y.M. 2013. Application of yeast Candida utilis to ferment Eisenia bicyclis for enhanced antibacterial effect. Applied Biochemistry and Biotechnology. 171: 569-582.

European Commission (EC), Guidance document describing the food categories in Part E of Annex II to Regulation (EC) No 1333/2008 on Food Additives [Accesses 2 march 2020] 2014. Available in: https://ec.europa.eu/food/sites/ food/files/safety/docs/fs_food-improvement-agents_ guidance_1333-2008_annex2.pdf

Faustman, C., Sun, Q., Mancini, R. and Suman, S.P. 2010. Myoglobin and lipid oxidation interactions: Mechanistic bases and control. Meat science. 86: 86-94.

Food and Drug Administration (FDA), Overview of food ingredients, additives \& colors [Accesses 2 march 2020] 2004. Available in: https://www.fda.gov/food/food-ingredientspackaging/overview-food-ingredients-additives-colors

Food and Drug Administration (FDA), Microbiological considerations for antimicrobial food additive submissions [Accesses 2 march 2020] 2008. Available in: https://www.fda. gov/media/83078/download 
Food and Agriculture Organization of the United Nations (FAO), The future of food and agriculture, trends and challenges [Accesses 2 march 2020] 2017. Available in: http://www.fao. org/3/a-i6583e.pdf

Food and Agriculture Organization of the United Nations (FAO), Codex alimentarius, norma general para los aditivos alimentarios [Accesses 2 march 2020] 2018. Available: http:// www.fao.org/gsfaonline/docs/CXS_192s.pdf

Hammad, H.H.M., Jin, G., Ma, M., Khalifa, I., Shukat, R., Elkhedir, A.E., Zeng, Q. and Noman, A.E. 2020. Comparative characterization of proximate nutritional compositions, microbial quality, and safety of camel meat in relation to mutton, beef, and chicken. LWT-Food Science and Technology. 118: 108714.

He, Y. and Shahidi, F. 1997. Antioxidant activity of green tea and its catechins in a fish meat model system. Journal of Agricultural and Food Chemistry. 45: 4262-4266.

Hernández-Carlos, B., Santos-Sánchez, N.F., Salas-Coronado, R., Villanueva-Cañongo, C. and Guadarrama-Mendoza, P.C. 2019. Antioxidant compounds from agro-industrial residue. In Antioxidants. IntechOpen (Ed). DOI: 10.5772/ intechopen.85184.

Hölker, U., Höfer, M. and Lenz, J. (2004). Biotechnological advantages of laboratory-scale solid-state fermentation with fungi. Applied Microbiology and Biotechnology. 64: 175-186.

Huang, W., Niu, H., Li, Z., Lin, W., Gong, G. and Wang, W. 2007. Effect of ellagitannin acyl hydrolase, xylanase and cellulase on ellagic acid production from cups extract of valonia acorns. Process Biochemistry. 42: 1291-1295.

Huang, Y.C., Chen, Y.F., Chen, C.Y., Chen, W.L., Ciou, Y.P., Liu, W.H. and Yang, C.H. 2011. Production of ferulic acid from lignocellulolytic agricultural biomass by Thermobifida fusca thermostable esterase produced in Yarrowia lipolytica transformant. Bioresource Technology. 102: 8117-8122.

Jiang, J. and Xiong, Y.L. 2016. Natural antioxidants as food and feed additives to promote health benefits and quality of meat products: A review. Meat Science. 120: 107-117.

Karastogiannidou, C. 1999. Effects of onion quercetin on oxidative stability of cook-chill chicken in vacuum-sealed containers. Journal of Food Science. 64: 978-981.

Łata, B., Trampczynska, A. and Paczesna, J. 2009. Cultivar variation in apple peel and whole fruit phenolic composition. Scientia Horticulturae. 121: 176-181.

Lokeshwari, N. and Reddy, S. 2010. Microbiological production of gallic acid by a mutant strain of Aspergillus oryzae using cashew husk. Pharmacophore. 1: 112-122.

Ma, T.W., Lai, Y. and Yang, F.C. 2014. Enhanced production of triterpenoid in submerged cultures of Antrodia cinnamomea with the addition of citrus peel extract. Bioprocess and Biosystems Engineering. 37: 2251-2261.

Machado, E.M., Rodriguez-Jasso, R.M., Teixeira, J.A. and Mussatto, S.I. 2012. Growth of fungal strains on coffee industry residues with removal of polyphenolic compounds. Biochemical Engineering Journal. 60: 87-90.

Maqsood, S., Abushelaibi, A., Manheem, K., Al Rashedi, A. and Kadim, I.T. 2015. Lipid oxidation, protein degradation, microbial and sensorial quality of camel meat as influenced by phenolic compounds. LWT-Food Science and Technology. 63: 953-959.
Marković, Z., Milenković, D., Đorović, J., Marković, J.M.D., Stepanić, V., Lučić, B. and Amić, D. 2012. PM6 and DFT study of free radical scavenging activity of morin. Food Chemistry. 134: 1754-1760

Moulehi, I., Bourgou, S., Ourghemmi, I. and Tounsi, M.S. 2012. Variety and ripening impact on phenolic composition and antioxidant activity of mandarin (Citrus reticulate Blanco) and bitter orange (Citrus aurantium L.) seeds extracts. Industrial Crops and Products. 39: 74-80.

Norma Oficial Mexicana NOM-122-SSA1-1994 (NOM), Bienes y servicios. Productos de la carne. Productos cárnicos curados y cocidos, y curados emulsionados y cocidos. Especificaciones sanitarias [Accesses 2 march 2020] 1994. Available in: http:// www.salud.gob.mx/unidades/cdi/nom/122ssa14.html

Norma Oficial Mexicana NOM-251-SSA1-2009 (NOM). Prácticas de higiene para el procesamiento de alimentos, bebidas o suplementos alimenticios [Accesses 2 march 2020] 2009. Available in: http://www.dof.gob.mx/normasOficiales/3980/ salud/salud.htm

Norma Oficial Mexicana NOM-213-SSA1-2002 (NOM), Productos y servicios. Productos cárnicos procesados. Especificaciones sanitarias. Métodos de prueba [Accesses 2 march 2020] 2002. Available in: http://www.salud.gob.mx/unidades/cdi/ nom/213ssa102.html

Onyeneho, S.N. and Hettiarachchy, N S. 1993. Antioxidant activity, fatty acids and phenolic acids compositions of potato peels. Journal of the Science of Food and Agriculture. 62: 345-350.

Orphanides, A., Goulas, V. and Gekas, V. 2013. Effect of drying method on the phenolic content and antioxidant capacity of spearmint. Czech Journal of Food Sciences. 31: 509-513.

Pandey, A. 2003. Solid-state fermentation. Biochemical Engineering Journal. 13: 81-84.

Papaspyridi, L.M., Aligiannis, N., Topakas, E., Christakopoulos, P., Skaltsounis, A.L. and Fokialakis, N. 2012. Submerged fermentation of the edible mushroom Pleurotus ostreatus in a batch stirred tank bioreactor as a promising alternative for the effective production of bioactive metabolites. Molecules. 17: 2714-2724.

Peanparkdee, M. and Iwamoto, S. 2019. Bioactive compounds from by-products of rice cultivation and rice processing: Extraction and application in the food and pharmaceutical industries. Trends in Food Science \& Technology. 86: 109117.

Pinelo, M., Tress, A.G., Pedersen, M., Arnous, A. and Meyer, A.S. 2007. Effect of cellulases, solvent type and particle size distribution on the extraction of chlorogenic acid and other phenols from spent coffee grounds. American Journal of Food and Technology. 2: 641-651.

Poljsak, B., Šuput, D. and Milisav, I. 2013. Achieving the balance between ROS and antioxidants: when to use the synthetic antioxidants. Oxidative medicine and Cellular Longevity. 1: $1-11$.

Pronyk, C. and Mazza, G. 2009. Design and scale-up of pressurized fluid extractors for food and bioproducts. Journal of Food Engineering. 95: 215-226.

Quirós-Sauceda, A. E., Palafox, H., Robles-Sánchez, R. M. and González-Aguilar, G. A. 2011. Interacción de compuestos fenólicos y fibra dietaría: capacidad antioxidante y biodisponibilidad. Biotecnia. 13: 3-11. 
Ramírez-Rojo, M.I., Vargas-Sánchez, R.D., del MarTorres-Martínez, B., Torrescano-Urrutia, G.R. and Sánchez-Escalante, A. 2018. Extractos de hojas de plantas para conservar la calidad de la carne y los productos cárnicos frescos. Revisión. Biotecnia. 20: $155-164$.

Ravichandran, M., Hettiarachchy, N.S., Ganesh, V., Ricke, S.C. and Singh, S. 2011. Enhancement of antimicrobial activities of naturally occurring phenolic compounds by nanoscale delivery against Listeria monocytogenes, Escherichia coli 0157: H7 and Salmonella typhimurium in broth and chicken meat system. Journal of Food Safety. 31: 462-471.

Razak, D.L.A., Rashid, N.Y.A., Jamaluddin, A., Sharifudin, S.A., Kahar, A.A. and Long, K. 2017. Cosmeceutical potentials and bioactive compounds of rice bran fermented with single and mix culture of Aspergillus oryzae and Rhizopus oryzae. Journal of the Saudi Society of Agricultural Sciences. 16: 127-134.

Rico, X., Gullón, B., Alonso, J.L. and Yáñez, R. 2020. Recovery of high value-added compounds from pineapple, melon, watermelon, and pumpkin processing by-products: an overview. Food Research International. 109086.

Rodríguez-Carpena, J.G., Morcuende, D., Andrade, M.J., Kylli, P. and Estévez, M. 2011. Avocado (Persea americana Mill.) phenolics, in vitro antioxidant and antimicrobial activities, and inhibition of lipid and protein oxidation in porcine patties. Journal of Agricultural and Food Chemistry. 59: 5625-5635.

Rodríguez Vaquero, M.J., Aredes Fernandez, P.A., Manca de Nadra, M.C. and Strasser de Saad, A.M. 2010. Phenolic compound combinations on Escherichia coli viability in a meat system. Journal of Agricultural and Food Chemistry. 58: 6048-6052.

Rodríguez Vaquero, M.J., Aredes Fernández, P.A., de Nadra, M. and Cristina, M. 2011. Effect of phenolic compound mixtures on the viability of Listeria monocytogenes in meat model. Food Technology and Biotechnology. 49: 83-88.

Sadh, P.K., Chawla, P. and Duhan, J.S. 2018. Fermentation approach on phenolic, antioxidants and functional properties of peanut press cake. Food Bioscience. 22: 113120.

Santana-Méridas, O., González-Coloma, A. and Sánchez-Vioque, R. 2012. Agricultural residues as a source of bioactive natural products. Phytochemistry Reviews. 11: 447-466.

Seth, M. and Chand, S. 2000. Biosynthesis of tannase and hydrolysis of tannins to gallic acid by Aspergillus awamorioptimisation of process parameters. Process Biochemistry. 36: 39-44.

Shahidi, F., Wanasundara, P.K.J.P.D. and Hong, C. 1992. Antioxidant activity of phenolic compounds in meat model systems. In: Phenolic compounds in foods and their effects on health. Ho, C.-T., Lee, C.Y. and Huang, M.-T. (Eds.). ACS Symposium Series 506, American Chemical Society, Washington, DC. pp. 214-222.

Shahidi, F., Yang, Z. and Saleemi, Z.O. 1993. Stabilization of meat lipids with flavonoids and flavonoid-related compounds. Journal of Food Lipids. 1: 69-78.

Shin, H.Y., Kim, S.M., Lee, J.H. and Lim, S.T. 2019. Solid-state fermentation of black rice bran with Aspergillus awamori and Aspergillus oryzae: effects on phenolic acid composition and antioxidant activity of bran extracts. Food Chemistry. 272: 235-241.
Sousa, B.A. and Correia, R.T.P. 2012. Phenolic content, antioxidant activity and antiamylolytic activity of extracts obtained from bioprocessed pineapple and guava wastes. Brazilian Journal of Chemical Engineering. 29: 25-30.

Spigno, G., Tramelli, L. and De Faveri, D.M. 2007. Effects of extraction time, temperature and solvent on concentration and antioxidant activity of grape marc phenolics. Journal of Food Engineering. 81: 200-208.

Sova, M. 2012. Antioxidant and antimicrobial activities of cinnamic acid derivatives. Mini-Reviews in Medicinal Chemistry. 12: 749-767.

Stojković, D., Petrović, J., Soković, M., Glamočlija, J., KukićMarković, J. and Petrović, S. 2013. In situ antioxidant and antimicrobial activities of naturally occurring caffeic acid, $p$-coumaric acid and rutin, using food systems. Journal of the Science of Food and Agriculture. 93: 3205-3208.

United States Department of Agriculture (USDA), Additives in Meat and Meat Products [Accesses 2 march 2020] 2015. Available in: https://www.fsis.usda.gov/wps/portal/fsis/ topics/food-safety-education/get-answers/food-safetyfact-sheets/food-labeling/additives-in-meat-and-poultryproducts/additives-in-meat-and-poultry-products

Valdez-Morales, M., Espinosa-Alonso, L.G., Espinoza-Torres, L.C., Delgado-Vargas, F. and Medina-Godoy, S. 2014. Phenolic content and antioxidant and antimutagenic activities in tomato peel, seeds, and by-products. Journal of Agricultural and Food Chemistry. 62: 5281-5289.

Vattem, D.A. and Shetty, K. 2002. Solid-state production of phenolic antioxidants from cranberry pomace by Rhizopus oligosporus. Food Biotechnology. 16: 189-210.

Vermerris, W. and Nicholson, R. 2008. Families of phenolic compounds and means of classification. In: Phenolic compound biochemistry. Springer (ed.), pp 1-34. Switzerland AG.

Wang, S.L., Hsiao, W.J. and Chang, W.T. 2002. Purification and characterization of an antimicrobial chitinase extracellularly produced by Monascus purpureus CCRC31499 in a shrimp and crab shell powder medium. Journal of Agricultural and Food Chemistry. 50: 2249-2255.

Wen, Y.L., Yan, L.P. and Chen, C.S. 2013. Effects of fermentation treatment on antioxidant and antimicrobial activities of four common Chinese herbal medicinal residues by Aspergillus oryzae. Journal of Food and Drug Analysis. 21: 219-226.

Wijngaard, H., Hossain, M.B., Rai, D.K. and Brunton, N. 2012. Techniques to extract bioactive compounds from food byproducts of plant origin. Food Research International. 46: 505-513.

Xie, C.Y., Gu, Z.X., You, X., Liu, G., Tan, Y. and Zhang, H. 2010. Screening of edible mushrooms for release of ferulic acid from wheat bran by fermentation. Enzyme and Microbial Technology. 46: 125-128.

$\mathrm{Xu}, \mathrm{X}$. and Zhu, J. 2011. Enhanced phenolic antioxidants production in submerged cultures of Inonotus obliquus in a ground corn stover medium. Biochemical Engineering Journal 58: 103-109.

Xu, X.Q., Hu, Y. and Zhu, L.H. 2014. The capability of Inonotus obliquus for lignocellulosic biomass degradation in peanut shell and for simultaneous production of bioactive polysaccharides and polyphenols in submerged fermentation. Journal of the Taiwan Institute of Chemical Engineers. 45: 2851-2858. 
Xu, X., Shen, M. and Quan, L. 2015. Stimulatory agents simultaneously improving the production and antioxidant activity of polyphenols from Inonotus obliquus by submerged fermentation. Applied Biochemistry and Biotechnology. 176: 1237-1250.

Yang, F.C. and Liau, C B. 1998. The influence of environmental conditions on polysaccharide formation by Ganoderma lucidum in submerged cultures. Process Biochemistry. 33: 547-553.
Yang, F.C., Ma, T.W. and Chuang, Y.T. 2012. Medium modification to enhance the formation of bioactive metabolites in shake flask cultures of Antrodia cinnamomea by adding citrus peel extract. Bioprocess and Biosystems Engineering. 35: 12511258.

Zhu, L. and $\mathrm{Xu}, \mathrm{X}$. 2013. Stimulatory effect of different lignocellulosic materials for phenolic compound production and antioxidant activity from Inonotus obliquus in submerged fermentation. Applied Biochemistry and Biotechnology. 169: 2138-2152. 